\title{
INTERVENÇÃO PSICOMOTORA NO DESENVOLVIMENTO INFANTIL: UMA REVISÃO INTEGRATIVA
}

\author{
Psychomotor intervention in child development: an integrative review \\ Intervención psicomotora para el desarrollo infantil: una revisión integrativa
}

\author{
Kátia Virginia Viana Cardoso (iD \\ Universidade Federal do Ceará - UFC - Fortaleza (CE) - Brasil
}

Sarah Amaral Lima (iD

Universidade Federal do Ceará - UFC - Fortaleza (CE) - Brasil

\section{RESUMO}

Objetivo: Compreender os efeitos da intervenção psicomotora frente ao atraso do desenvolvimento neuropsicomotor de crianças. Métodos: O presente estudo trata-se de uma revisão integrativa da literatura científica. Para a realização da pesquisa, artigos foram buscados nas seguintes bases de dados: PubMed, PEDro (Physiotherapy Evidence Database), BVS (Biblioteca Virtual em Saúde), Portal de Periódicos CAPES e Web of Science, no período de dezembro de 2018 a janeiro de 2019, e houve consulta ao Research gate no mês de janeiro de 2019. Resultados: Foram encontrados 867 artigos, dos quais se excluíram 142 devido à falta de conformidade aos critérios de inclusão e 33 foram lidos na íntegra, sendo o estudo composto de 7 artigos selecionados de acordo com os critérios, nos quais se observou que a psicomotricidade contribui positivamente para o desenvolvimento da criança. Conclusão: A contribuição da intervenção psicomotora estruturada, apesar da heterogeneidade de protocolos adotados, apresenta resultados positivos no processo de desenvolvimento neuropsicomotor na infância, sendo um recurso passível de utilização para recuperar ou prevenir atrasos no desenvolvimento infantil.

Descritores: Desenvolvimento Infantil; Intervenção Precoce (Educação); Deficiências do Desenvolvimento.

\section{ABSTRACT}

Objective: Understand the effects of psychomotor intervention in children with neuropsychomotor developmental delay. Methods: This study is an integrative review of the scientific literature. For the research, articles were searched in the following databases: PubMed, PEDro (Physiotherapy Evidence Database), VHL (Virtual Health Library). CAPES Journals Portal and Web of Science, from December 2018 to January 2019, and the Research gate were consulted in January 2019. Results: A total of 867 articles were found, of which 142 were excluded due to non-compliance with the inclusion criteria and 33 were read in full. The study consisted of 7 articles selected according to the criteria, which showed that psychomotricity contributes positively to child development. Conclusion: The contribution of structured psychomotor intervention, despite the heterogeneity of protocols adopted, has positive results in the neuropsychomotor development process in childhood, being a usable resource to recover or prevent delays in child development.

Descriptors: Child development; Early Intervention (Education); Developmental Disabilities.

\section{RESUMEN}

Objetivo: Comprender los efectos de la intervención psicomotora frente el retraso del desarrollo neuropsicomotor de niños. Métodos: El presente estudio se trata de una revisión integrativa de la literatura científica. Para la realización de la investigación se buscó artículos en las bases de datos PubMed, PEDro (Physiotherapy Evidence Database), BVS (Biblioteca Virtual en Salud), Portal de Periódicos CAPES y Web of Science en el periodo entre diciembre de 2018 y enero de 2019 y la consulta en el Research gate en el mes de enero de 2019. Resultados: Se han encontrado 867 artículos de los cuales 142 han sido excluidos por el no cumplimiento de los criterios de inclusión. Se han leído 33 artículos completos de los cuales 7 han sido elegidos según los criterios de los cuales se ha observado que la psicomotricidad ha contribuido de manera positiva para el desarrollo del niño. Conclusión: Pese la heterogeneidad de los protocolos adoptados, la contribución de la intervención psicomotora estructurada

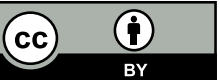


presenta resultados positivos en el proceso del desarrollo neuropsicomotor en la infancia y es un recurso pasible de utilización para recuperar o prevenir los retrasos del desarrollo infantil.

Descriptores: Desarrollo Infantil; Intervención Precoz (Educación); Discapacidades del Desarrollo.

\section{INTRODUÇÃO}

O desenvolvimento infantil tem seu início na vida intrauterina e envolve os processos de maturação neurológica, aquisição das habilidades motoras, cognitivas, sociais e emocionais, além do crescimento físico, e está sujeito às influências dos fatores biológicos e ambientais. Com o nascimento, inicia-se a vigilância aos domínios de funções indicativas do neurodesenvolvimento, que são: as funções sensoriais, psicomotoras, sociais, cognitivas, motoras finas e grosseiras, comunicação e linguagem; sendo esse monitoramento o preditor da necessidade de estimulação precoce para a promoção do desenvolvimento infantil ou intervenção precoce em casos de identificação de transtorno no neurodesenvolvimento( ${ }^{(1,2)}$.

A literatura científica apresenta diversos estudos que demonstram que os prejuízos decorrentes dos atrasos no desenvolvimento infantil são, em sua maioria, futuras preocupações sociais, pois ele está relacionado com a posterior evasão escolar, gravidez na adolescência e delitos juvenis, portanto, um ambiente inibidor, inadequado ou pouco estimulante poderá ocasionar repercussões negativas no neurodesenvolvimento da criança ${ }^{(3-6)}$.

A Psicomotricidade é uma ciência relativamente nova, com surgimento na França no início do século XX, entendida como campo transdisciplinar, que estuda e investiga as influências e relações entre o psiquismo e a motricidade emergentes da personalidade total, singular e evolutiva que caracteriza o ser humano, nas múltiplas e complexas manifestações biopsicossociais, afetivo-emocionais e psicosociocognitivas. A psicomotricidade apresenta sete elementos básicos: o esquema corporal, a tonicidade, a lateralidade, a estruturação espacial, a orientação temporal, o ritmo e o equilíbrio ${ }^{(7-9)}$.

Desse modo, a atividade psicomotora tem como objetivo utilizar o corpo, no sentido de perpassar o real, indo além, interagindo com o corpo que se move. Essa transcendência permite que o corpo não esteja apenas voltado para si com vistas à melhora física, mas, sim, uma busca por um espaço em que o simbolismo é permitido, sendo a expressão da mente pelo corpo ${ }^{(10-12)}$.

A utilização da psicomotricidade como intervenção precoce, realizada por profissionais da saúde, no estímulo ao desenvolvimento infantil, integra o motor e o psicológico, fazendo a importante relação entre mente e corpo, assim constituindo a base do desenvolvimento psicomotor. Portanto, o desenvolvimento neuropsicomotor é fundamental na vida da criança, porque, através da descoberta realizada por meio do seu corpo, dos movimentos e de tudo que está em seu ambiente, ela conseguirá organizar e conquistar seu espaço, desenvolver habilidades e emoções, aprendendo aos poucos a coordená-las de modo a buscar sua independência ${ }^{(10-12)}$.

Dessa forma, o esquema corporal e a imagem do corpo são campos do desenvolvimento humano, mostrando que o psiquismo influência as habilidades motoras, movimentos, posturas e o relacionamento do ser humano com o ambiente ${ }^{(10,11)}$. Assim, o estudo se justifica pela necessidade de prevenir ou recuperar atrasos no desenvolvimento neuropsicomotor durante a infância, uma vez que a condição se mostra prevalente e esses indivíduos, quando adentram a adolescência e a fase adulta, apresentam resultados negativos para a sociedade, além de provocarem o aumento da evasão escolar, da gravidez na adolescência, dos gastos em sistema de segurança e de saúde públicos ${ }^{(13)}$.

O objetivo dessa revisão é, portanto, compreender os efeitos da intervenção psicomotora frente ao atraso do desenvolvimento neuropsicomotor de crianças.

\section{MÉTODOS}

Estudo de revisão integrativa da literatura científica realizado a partir da seguinte pergunta norteadora: Quais os efeitos da intervenção psicomotora em crianças com atraso no desenvolvimento neuropsicomotor? Por conseguinte, buscou-se na literatura vigente evidências sobre a utilização da intervenção psicomotora como tratamento de crianças com atraso no desenvolvimento neuropsicomotor, com o objetivo principal de identificar os protocolos de intervenção psicomotora eficientes e compreender suas repercussões no atraso do desenvolvimento infantil. 
A hipótese formulada foi de que, para prevenir ou recuperar atrasos no desenvolvimento infantil, a intervenção psicomotora está consolidada e evidenciada cientificamente e que os estudos provavelmente apontarão resultados favoráveis, apesar de existir heterogeneidade nas intervenções utilizadas.

Para tanto, realizaram-se buscas por artigos publicados nas seguintes bases de dados: LILACS, SciELO, PubMed, Portal de Periódicos Capes, Physiotherapy Evidence Datebases (PEDro) e Web of Science, durante os meses de dezembro de 2018 e janeiro de 2019, correspondendo ao período que se finalizou a pesquisa. A busca em português baseou-se nos descritores em Ciências da Saúde (DeCS) da Biblioteca Virtual em Saúde, e os descritores em inglês foram baseados no Medical Subject Headings (MeSH), com exceção do termo psicomotricidade, no inglês psychomotricity, que não possui correspondentes no DeCS ou MeSH. Os descritores utilizados na busca em português foram: desenvolvimento infantil, deficiências do desenvolvimento, intervenção precoce, psicomotricidade e fisioterapia. Em inglês, respectivamente: child development, child development disorder, early intervention, psychomotricity e physical therapy modalities (Quadro I). Foram acrescentados ao estudo uma consulta ao Research Gate no mês de janeiro de 2019, um estudo publicado na Revista Nuances da Educação, e um estudo publicado nos Anais do Congresso de Iniciação Científica Estágio e Docência do Campus Formosa.

Desse modo, iniciou-se a busca para a identificação e captação máxima dos estudos disponíveis relacionados ao objetivo deste artigo e que atendessem aos critérios de inclusão e exclusão previamente estabelecidos. Em seguida, foi aplicada a escala PEDro para avaliar a qualidade metodológica e a descrição estatística dos artigos selecionados para esta revisão integrativa. Nessa escala a pontuação varia de 0 a 10, com base em 11 critérios (Critérios de inclusão especificados, alocação aleatória, sigilo na alocação, similaridade entre grupos no pré-tratamento, sujeitos cegos, terapeutas cegos, examinadores cegos, follow-up de pelo menos $85 \%$ dos participantes, intenção de tratar, comparações estatísticas entre os grupos e relato das medidas de variabilidade) $)^{(14)}$.

Incluíram-se estudos nos idiomas inglês e/ou português que tivessem como população-alvo crianças de 0 a 6 anos, com atraso no desenvolvimento neuropsicomotor, que utilizaram a intervenção neuropsicomotora/ psicomotora em seu estudo, e artigos dos últimos 10 anos (2008 a 2018). Foram excluídos: estudos que não especificassem no texto a metodologia utilizada; estudos que tratassem dos atrasos psicomotores em crianças atípicas, com disfunções neuromotoras e ortopédicas, embora título e resumo correspondessem ao objeto do estudo; duplicatas; revisões e cartas.

\begin{tabular}{|ll|}
\hline "Desenvolvimento Infantil" & 1) “Child development" \\
\hline "Deficiências do desenvolvimento" & 2) “Child development disorder" \\
\hline "Intervenção precoce" & 3) "Early intervention" \\
\hline "Fisioterapia" & 4) “Physical therapy modalities" \\
\hline "Psicomotricidade" & 5) "Psychomotricity" \\
\hline $\begin{array}{l}\text { Cruzamentos: (1 AND } 3 \text { AND 4) OR (1 AND 4 AND 5) OR (1 AND 3) OR ( } 1 \text { AND 4) OR (1 AND 5) OR (2 AND } 3 \text { AND 4) } \\
\text { OR (2 AND 4 AND 5) OR (2 AND 3) OR (2 AND 4) OR (2 AND 5). }\end{array}$ \\
\hline
\end{tabular}

Quadro I. Termos e cruzamentos utilizados nas buscas eletrônicas.

\section{RESULTADOS}

Os seguintes artigos foram identificados: BVS ( $n=265)$; Periódico CAPES $(n=156) ;$ PubMed $(n=164)$; Base PEDro ( $n=6)$, Web of Science ( $n=266)$, Researchgate $(n=11)$, um estudo da Revista Nuances da Educação, e uma publicação em Anais, porém, após a leitura de títulos e resumos, selecionaram-se somente 33 estudos para leitura na íntegra, dos quais restaram 7 artigos, que respeitavam os critérios de inclusão e exclusão previamente descritos, além de atenderem aos princípios preconizados no desenvolvimento de uma revisão integrativa.

O fluxograma apresenta como foram coletados os estudos incluídos na pesquisa (Figura 1). Os estudos selecionados para discussão neste artigo de revisão estão descritos no Quadro Il e classificados de acordo com a pontuação da escala PEDro ${ }^{(14)}$, apresentando uma pontuação que variou de 4 a 8 pontos, e a qualidade metodológica desses estudos está apresentada na Tabela I. 


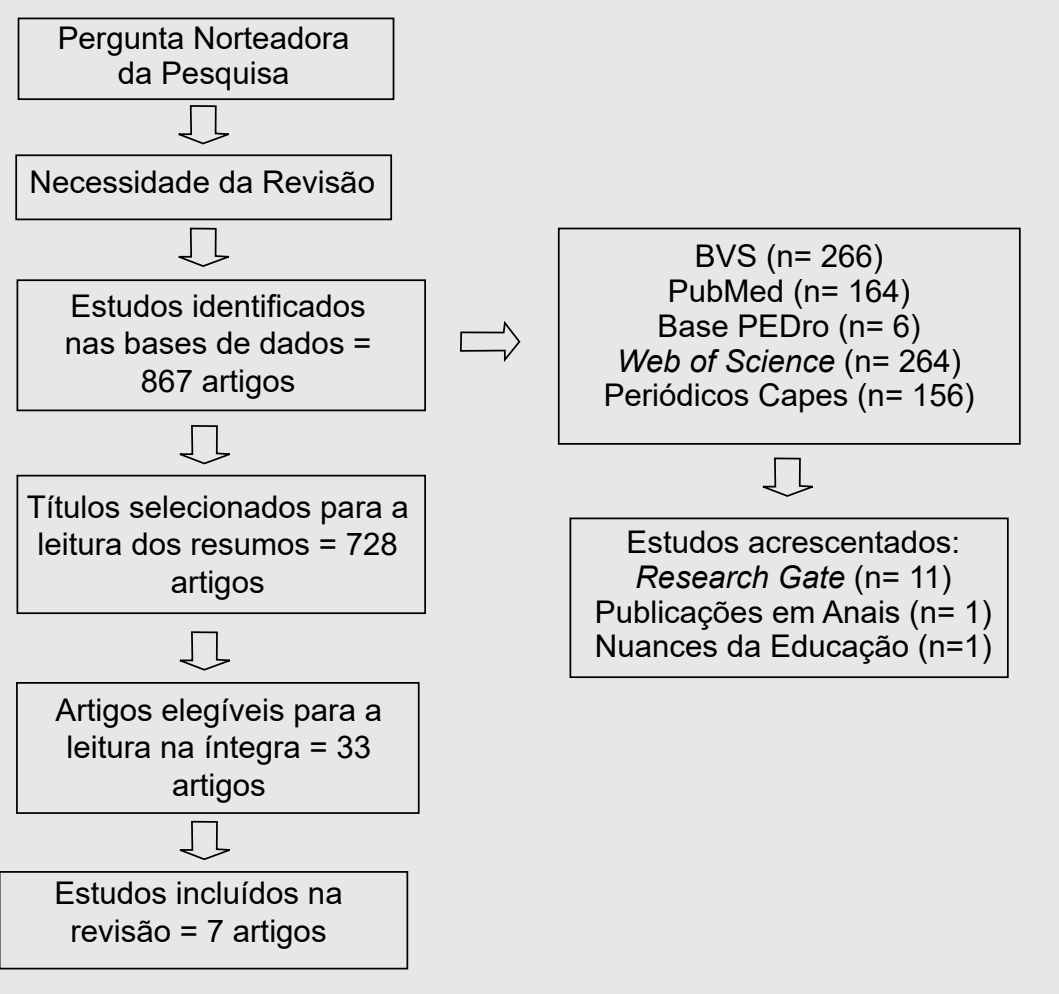

Figura 1 - Fluxograma de identificação e seleção de artigos para a revisão integrativa.

Quadro II - Caracterização dos artigos segundo autor/ano, objetivo, abordagem metodológica, intervenção, resultados e conclusão.

\begin{tabular}{|c|c|c|c|c|c|}
\hline Autor/ ano & Objetivo & $\begin{array}{l}\text { Abordagem } \\
\text { metodológica }\end{array}$ & $\begin{array}{l}\text { Intervenção } \\
\text { estudada }\end{array}$ & Resultados & Conclusão \\
\hline $\begin{array}{l}\text { Soejima et } \\
\text { al. } 2012^{(15)}\end{array}$ & $\begin{array}{l}\text { Verificar se um } \\
\text { programa de } \\
\text { intervenção } \\
\text { precoce tem efeitos } \\
\text { positivos sobre o } \\
\text { desenvolvimento } \\
\text { neuromotor de } \\
\text { crianças entre } 0 \text { e } 3 \\
\text { anos que estudam em } \\
\text { creche pública. }\end{array}$ & $\begin{array}{l}63 \text { crianças, de } 0 \text { a } 36 \\
\text { meses, distribuídas em } \\
\text { três turmas. As crianças } \\
\text { foram avaliadas por } \\
\text { meio das Escalas } \\
\text { Mental e Motora de } \\
\text { Desenvolvimento Infantil } \\
\text { Bayley II e o programa } \\
\text { de intervenção } \\
\text { precoce foi elaborado } \\
\text { individualmente para } \\
\text { cada bebê. }\end{array}$ & \begin{tabular}{|} 
Foram selecionadas \\
20 crianças \\
com atraso no \\
desenvolvimento \\
e a intervenção \\
ocorreu em 1 mês, \\
de segunda à sexta- \\
feira, com duração de \\
20 minutos.
\end{tabular} & $\begin{array}{c}\text { Foi evidenciada } \\
\text { progressão no } \\
\text { desenvolvimento das } \\
\text { crianças participantes } \\
\text { da intervenção, } \\
\text { quando comparados } \\
\text { ao Bayley II do pré e } \\
\text { pós-intervenção. }\end{array}$ & $\begin{array}{l}\text { Foi verificada a } \\
\text { viabilidade da } \\
\text { intervenção precoce } \\
\text { nas creches } \\
\text { públicas, porém } \\
\text { novas pesquisas } \\
\text { devem ser } \\
\text { realizadas visando a } \\
\text { encontrar sugestões } \\
\text { e respostas de } \\
\text { formas pertinentes } \\
\text { de atendimento. }\end{array}$ \\
\hline $\begin{array}{l}\text { Santos et al. } \\
2016^{(16)}\end{array}$ & $\begin{array}{c}\text { Verificar os efeitos de } \\
\text { duas diferentes } \\
\text { intervenções } \\
\text { psicomotoras no } \\
\text { desenvolvimento } \\
\text { socioemocional e } \\
\text { de autoconceito da } \\
\text { criança. }\end{array}$ & $\begin{array}{c}\text { Participaram } 46 \\
\text { crianças da educação } \\
\text { pré-escolar, com } \\
4,1 \pm 0,8 \text { anos. Destas } \\
46 \text { crianças, } 17 \\
\text { pertenciam ao grupo } \\
\text { de psicomotricidade } \\
\text { relacional, } 17 \text { ao grupo } \\
\text { de psicomotricidade } \\
\text { funcional e } 12 \text { ao grupo } \\
\text { de controle. }\end{array}$ & $\begin{array}{c}\text { Os } 46 \text { participantes } \\
\text { ficaram divididos em } \\
\text { três grupos (GE, GD } \\
\text { e GC). Os grupos GE } \\
\text { e GD receberam } 24 \\
\text { sessões, } 2 \text { vezes por } \\
\text { semana, ao longo de } \\
3 \text { meses. }\end{array}$ & $\begin{array}{l}\text { Foram encontradas } \\
\text { diferenças } \\
\text { estatisticamente } \\
\text { significativas na } \\
\text { psicomotricidade } \\
\text { relacional, entre o } \\
\text { pré e o pós-teste, as } \\
\text { quais foram } \\
\text { observadas na } \\
\text { diminuição dos } \\
\text { problemas de } \\
\text { comportamento. }\end{array}$ & $\begin{array}{l}\text { O método utilizado } \\
\text { na psicomotricidade } \\
\text { relacional permite } \\
\text { a espontaneidade } \\
\text { da criança como } \\
\text { eixo da intervenção. } \\
\text { Desse modo, } \\
\text { os ganhos de } \\
\text { habilidades } \\
\text { cognitivas, motoras, } \\
\text { sociais e emocionais } \\
\text { parecem ser mais } \\
\text { favorecidos. }\end{array}$ \\
\hline
\end{tabular}




\begin{tabular}{|c|c|c|c|c|c|}
\hline $\begin{array}{l}\text { Costa et al. } \\
2016^{(17)}\end{array}$ & $\begin{array}{l}\text { Verificar a influência } \\
\text { da Educação Física } \\
\text { no desenvolvimento } \\
\text { psicomotor de alunos } \\
\text { com cinco anos do } \\
\text { ensino pré-escolar. }\end{array}$ & $\begin{array}{c}\text { Participaram do estudo } \\
126 \text { alunos portugueses } \\
\text { (71 do sexo feminino e } \\
55 \text { do sexo masculino) } \\
\text { de } 5 \text { anos. Os alunos da } \\
\text { amostra foram divididos } \\
\text { em dois grupos: o grupo } \\
\text { controle (GC } n=64 \text { ) e o } \\
\text { grupo experimental (GE } \\
n=62 \text { ). }\end{array}$ & $\begin{array}{l}\text { O grupo experimental } \\
\text { (GE) recebeu } \\
\text { intervenção durante } \\
24 \text { semanas, com } 2 \\
\text { sessões semanais, } \\
\text { de } 45 \text { minutos, } \\
\text { durante as aulas de } \\
\text { Educação Física. }\end{array}$ & $\begin{array}{c}\text { Os resultados } \\
\text { demonstraram que } \\
\text { ambos os grupos } \\
\text { evoluíram o seu } \\
\text { perfil psicomotor, } \\
\text { no entanto essa } \\
\text { evolução foi sempre } \\
\text { superior no GE. Não } \\
\text { houve variações } \\
\text { significativas quanto } \\
\text { ao sexo. }\end{array}$ & $\begin{array}{l}\text { A Educação Física } \\
\text { estruturada é } \\
\text { importante para o } \\
\text { desenvolvimento } \\
\text { psicomotor da } \\
\text { criança em idade } \\
\text { pré-escolar. }\end{array}$ \\
\hline $\begin{array}{l}\text { Ene et al. } \\
2016^{(18)}\end{array}$ & $\begin{array}{l}\text { Melhorar os } \\
\text { componentes da } \\
\text { psicomotricidade em } \\
\text { crianças com idade } \\
\text { entre } 4 \text { e } 6 \text { anos } \\
\text { através de atividades } \\
\text { lúdicas realizadas } \\
\text { dentro do quadro de } \\
\text { atividade física. }\end{array}$ & $\begin{array}{c}\text { A amostra foi composta } \\
\text { por } 96 \text { crianças, de } 4 \text { a } \\
6 \text { anos, estudantes de } \\
\text { uma escola primária na } \\
\text { Romênia. As crianças } \\
\text { foram divididas em três } \\
\text { grupos: } 4 \text { anos, } 5 \text { anos } \\
\text { e } 6 \text { anos. Foi aplicado } \\
\text { um teste de pré e } \\
\text { pósintervenção. }\end{array}$ & \begin{tabular}{|c|} 
As crianças \\
participaram de um \\
programa psicomotor, \\
com frequência de 2 \\
vezes por semana, \\
com duração de 40 \\
a 50 minutos, por um \\
período de um ano.
\end{tabular} & $\begin{array}{c}\text { O grupo de crianças } \\
\text { de } 4 \text { anos melhorou } \\
\text { significativamente a } \\
\text { orientação espacial } \\
\text { e consciência } \\
\text { corporal. As } \\
\text { crianças de } 5 \text { anos } \\
\text { não apresentaram } \\
\text { diferenças, enquanto } \\
\text { que das crianças } \\
\text { de } 6 \text { anos foram } \\
\text { adquiridas melhoras. }\end{array}$ & $\begin{array}{c}\text { O papel da } \\
\text { educação infantil } \\
\text { é essencial no } \\
\text { desenvolvimento da } \\
\text { criança. A escola } \\
\text { primária deve } \\
\text { incluir atividades } \\
\text { físicas (movimento } \\
\text { e jogos) centradas } \\
\text { na melhora } \\
\text { psicomotora. }\end{array}$ \\
\hline $\begin{array}{l}\text { Panceri et al. } \\
2017^{(19)}\end{array}$ & $\begin{array}{l}\text { Avaliar o impacto da } \\
\text { intervenção motora } \\
\text { no desenvolvimento } \\
\text { motor e cognitivo } \\
\text { de bebês de } 1 \text { a } 18 \\
\text { meses hospitalizados } \\
\text { por doenças } \\
\text { respiratórias. }\end{array}$ & $\begin{array}{c}\text { Estudo quase } \\
\text { experimental, com } 39 \\
\text { bebês hospitalizados, } \\
\text { conforme diagnóstico } \\
\text { médico, e ausentes de } \\
\text { sinais e sintomas de } \\
\text { doenças neurológicas } \\
\text { ou mentais previamente } \\
\text { diagnosticadas. }\end{array}$ & $\begin{array}{c}\text { Os bebês foram } \\
\text { divididos em } 2 \\
\text { grupos, sendo o GC } \\
(\mathrm{n}=10) \text { e } \mathrm{GI}(\mathrm{n}=12) . \\
\text { O GI participava } \\
\text { de um programa } \\
\text { de intervenção } \\
\text { cognitivomotora } \\
\text { diariamente, por } 30 \\
\text { minutos, até a alta } \\
\text { hospitalar. }\end{array}$ & \begin{tabular}{|c|} 
Foi observada uma \\
interação significativa \\
entre grupo x \\
tempo, nos escores \\
motores e cognitivos. \\
Ao comprar o \\
período pré e pós- \\
intervenção, o GI \\
apresentou melhoras \\
significativas \\
positivas.
\end{tabular} & $\begin{array}{c}\text { A intervenção } \\
\text { cognitivo-motora no } \\
\text { ambiente hospitalar } \\
\text { durante o tempo } \\
\text { de internação dos } \\
\text { bebês contribuiu de } \\
\text { forma positiva para } \\
\text { o desenvolvimento } \\
\text { cognitivo e motor. }\end{array}$ \\
\hline $\begin{array}{l}\text { Brandão et } \\
\text { al.2017(20) }\end{array}$ & $\begin{array}{l}\text { Abordar o aspecto } \\
\text { motor e as } \\
\text { brincadeiras no } \\
\text { desenvolvimento } \\
\text { infantil, segundo a } \\
\text { teoria psicogenética } \\
\text { de Jean Piaget. }\end{array}$ & $\begin{array}{l}\text { A amostra foi composta } \\
\text { de } 15 \text { crianças, de } \\
\text { ambos os sexos, entre } \\
2 \text { e } 3 \text { anos, de uma } \\
\text { escola pública de } \\
\text { Goiás. }\end{array}$ & $\begin{array}{l}\text { As crianças formaram } \\
\text { um grupo único, } \\
\text { e a intervenção } \\
\text { psicomotora foi } \\
\text { composta de } 10 \\
\text { sessões, com } \\
1 \text { encontro por } \\
\text { semana, com a } \\
\text { duração } 3 \text { horas e } 15 \\
\text { min cada. }\end{array}$ & $\begin{array}{c}\text { As atividades } \\
\text { trabalhadas } \\
\text { contribuíram para } \\
\text { o desenvolvimento } \\
\text { motor, a interação } \\
\text { social e a } \\
\text { autoconfiança. }\end{array}$ & $\begin{array}{c}\text { A brincadeira } \\
\text { possibilita a criança } \\
\text { o movimento e } \\
\text { a exploração do } \\
\text { espaço ao seu redor, } \\
\text { sendo essencial, } \\
\text { segundo a teoria } \\
\text { piagetiana, para a } \\
\text { passagem de cada } \\
\text { estágio. }\end{array}$ \\
\hline $\begin{array}{l}\text { Mas et al. } \\
2018^{(21)}\end{array}$ & $\begin{array}{c}\text { Mostrar como as } \\
\text { práticas habituais de } \\
\text { psicomotricidade a } \\
\text { partir dos } 12 \text { meses } \\
\text { de idade podem } \\
\text { elevar o } \\
\text { desenvolvimento de } \\
\text { crianças. }\end{array}$ & $\begin{array}{l}\text { Foram selecionadas } \\
26 \text { crianças, entre } \\
11 \text { a } 22 \text { meses, que } \\
\text { frequentavam uma } \\
\text { escola de educação } \\
\text { infantil em uma } \\
\text { província de Barcelona. }\end{array}$ & $\begin{array}{c}\text { O estudo foi dividido } \\
\text { em duas partes: } \\
1 \text { - treinamento de } \\
5 \text { meses com as } \\
\text { crianças divididas } \\
\text { em } 3 \text { grupos (G0, } \\
\text { G1 e G2); } 2-U m \\
\text { período de prática } \\
\text { psicomotora de } \\
23 \text { meses com as } \\
\text { crianças divididas } \\
\text { nos mesmos grupos. }\end{array}$ & \begin{tabular}{|c|} 
Os resultados obtidos \\
com as medidas \\
reunidas na fase \\
de treinamento, \\
e na fase prática \\
pós-final conclui que \\
a sistematização da \\
atividade psicomotora \\
tem influenciado \\
positivamente o \\
desenvolvimento \\
de crianças pré- \\
escolares.
\end{tabular} & $\begin{array}{c}\text { A psicomotricidade } \\
\text { é uma atividade } \\
\text { necessária na } \\
\text { primeira infância, } \\
\text { sendo uma atividade } \\
\text { divertida e relacional } \\
\text { que } \\
\text { contribui para o } \\
\text { desenvolvimento } \\
\text { ideal. }\end{array}$ \\
\hline
\end{tabular}


Tabela I - Classificação metodológica avaliada pela escala PEDro.

\begin{tabular}{|c|c|c|c|c|c|c|c|}
\hline & $\begin{array}{l}\text { Soejima et al. } \\
\qquad \mathbf{2 0 1 2}^{(15)}\end{array}$ & $\begin{array}{l}\text { Ene et al. } \\
2016^{(16)}\end{array}$ & $\begin{array}{l}\text { Santos et al. } \\
\qquad 2016^{(17)}\end{array}$ & $\begin{array}{c}\text { Costa et al. } \\
2016^{(18)}\end{array}$ & $\begin{array}{c}\text { anceri et al. } \\
2017^{(19)}\end{array}$ & $\begin{array}{l}\text { Brandão et al. } \\
\qquad 2017^{(20)}\end{array}$ & $\begin{array}{c}\text { mas et al. } \\
2018^{(21)}\end{array}$ \\
\hline $\begin{array}{l}\text { 1. Critérios de inclusão } \\
\text { especificados }\end{array}$ & Sim & Sim & Sim & Sim & Sim & - & Sim \\
\hline 2. Alocação aleatória & Não & Sim & Não & Sim & Sim & - & Não \\
\hline 3. Sigilo na alocação & Não & Não & Não & Sim & Sim & - & Não \\
\hline $\begin{array}{l}\text { 4. Similaridade entre } \\
\text { grupos no pré-tratamento }\end{array}$ & Sim & Não & Sim & Sim & Sim & - & Sim \\
\hline 5. Sujeitos cegos & Sim & Sim & Não & Sim & Sim & - & Sim \\
\hline 6. Terapeutas cegos & Não & Não & Não & Não & Não & - & Não \\
\hline 7. Examinadores cegos & Não & Não & Não & Não & Sim & - & Não \\
\hline $\begin{array}{l}\text { 8. Follow-up de pelo } \\
\text { menos } 85 \% \text { dos }\end{array}$ & & & & & & & \\
\hline participantes & Não & Não & Sim & Sim & Não & - & Não \\
\hline 9. Intenção de tratar & Sim & Sim & Sim & Sim & Sim & - & Sim \\
\hline $\begin{array}{l}\text { 10. Comparações } \\
\text { estatísticas entre os } \\
\text { grupos }\end{array}$ & Sim & Não & Sim & Sim & Sim & - & Não \\
\hline $\begin{array}{l}\text { 11. Relatos das medidas } \\
\text { de visibilidade }\end{array}$ & Não & Sim & Sim & Sim & Sim & - & Sim \\
\hline Total & 4 & 4 & 7 & 8 & - & - & 4 \\
\hline
\end{tabular}

\section{DISCUSSÃO}

Diante dos resultados obtidos, buscou-se discutir as semelhanças e divergências entre os achados dos estudos e confrontar com a literatura como as intervenções psicomotoras podem ser implementadas no desenvolvimento infantil, minimizando os atrasos no desenvolvimento neuropsicomotor da criança, promovendo a estimulação precoce, para que possíveis atrasos possam ser superados.

\section{A intervenção psicomotora no ambiente hospitalar}

O ambiente no qual a criança está inserida é capaz de influenciar seu desenvolvimento. Desse modo, a hospitalização é pontuada como um dos fatores que pode repercutir negativamente sobre o desenvolvimento infantil, pois, na maioria das vezes, a experiência é traumática e desagradável, além de existir restrições quanto ao espaço físico, iluminação, mobilidade, elevados estímulos sonoros e toques constantes ${ }^{(18)}$.

Assim sendo, um estudo com o objetivo de investigar o impacto da intervenção cognitivo-motora no desenvolvimento motor e cognitivo de bebês internados, decorrentes de patologias respiratórias, selecionou 39 crianças. Destas, 12 fizeram parte do grupo intervenção. A intervenção cognitivomotora acontecia diariamente, durante 30 minutos, sendo realizada na sala de recreação da unidade ou no próprio leito até o momento de alta hospitalar do participante. As atividades desenvolvidas consistiam na tentativa de oportunizar ao bebê experimentar novas habilidades motoras e cognitivas, ocorrendo da seguinte maneira: intervenções lúdicas, em que os 5 minutos de início eram utilizados para ambientação; em seguida 5 minutos na posição prona ou supina, destinada a realização de alongamentos, estímulo do rolar, perseguição visual e exploração de brinquedos; nos próximos 10 minutos era vivenciada a posição sentada; os 10 minutos finais eram utilizados para o favorecimento do deslocamento da criança. Como resultado analisado após aplicação da Alberta Infant Motor Scale (AIMS) e Escala Bayle III, no período de pré e pós-intervenção, o estudo sugere que esse tipo de intervenção durante o período de hospitalização do bebê contribui positivamente para o desenvolvimento infantil, protegendo a criança dos efeitos negativos da internação hospitalar por doenças respiratórias ${ }^{(19)}$.

Segundo aplicação da escala PEDro, o estudo obteve pontuação igual a 8, mostrando ser de boa qualidade metodológica, contudo verificamos que a falta de padronização no número de intervenções, o tamanho amostral pequeno, o cegamento dos terapeutas e a ausência de follow-up contribuem para que a pontuação não seja melhor. 


\section{A intervenção psicomotora na primeiríssima infância}

A psicomotricidade oportuniza a criança o brincar com os objetos em seu contexto, pois, através do movimento, inclui a interação entre neuromotor e funções psicológicas, contribuindo para o desenvolvimento neuropsicomotor da criança. Assim, a qualidade que o ambiente pode oferecer desde o seu nascimento tem impacto posterior em todas as etapas do desenvolvimento infantil ${ }^{(14,20)}$.

Outro estudo buscou verificar se um programa de intervenção precoce tem efeitos positivos sobre o desenvolvimento psicomotor de crianças entre 0 e 3 anos que permaneciam em tempo integral em uma creche pública. $O$ programa de 1 mês consistiu, então, no planejamento individual de atividades lúdicas para as 20 crianças do grupo intervenção, com duração de 20 minutos, uma vez por semana. Os resultados obtidos evidenciaram progressão no desenvolvimento das crianças que apresentaram defasagem e que participaram do programa de intervenção quando comparadas a crianças com atraso no desenvolvimento que não participaram da intervenção. Assim, segundo os resultados comparados da aplicação das Escalas Mental e Motora do Desenvolvimento Infantil Bayle II, no período pré e pósintervenção, subsidiam a utilização de programas de intervenção precoce nas creches públicas ${ }^{(15)}$.

A aplicação da escala PEDro classifica o estudo como de qualidade metodológica ruim, sendo sua pontuação igual a 4, pois detalhes como cegamento dos terapeutas e examinadores, não detalhamento das intervenções realizadas, a não realização de follow-up e o não relato das medidas de variabilidade contribuíram para uma pontuação ruim segundo a escala.

Dentro deste contexto de primeiríssima infância, outro estudo mostrou como as práticas de psicomotricidade a partir dos 12 meses podem contribuir para desenvolvimento neuropsicomotor de crianças. Os participantes da intervenção foram divididos em 2 grupos, G1 com 8 crianças que receberam atendimento 1 vez por semana, e G2 com 7 crianças que recebiam atendimento 2 vezes por semana. A intervenção aconteceu durante 23 meses, com duração de 45 minutos por sessão, dividida em três momentos curtos. O primeiro momento consistia no acolhimento do grupo; em seguida, jogos livres que envolviam, sobretudo, a motricidade; e o último momento, de retorno à calma. Mediante análises dos resultados obtidos com a aplicação do teste Merill-Palmer-R no período pré e pósintervenção, evidenciou-se que a psicomotricidade é necessária nessa fase do desenvolvimento, podendo contribuir para a identificação de problemas no desenvolvimento de habilidades no período pré-escolar. Portanto, a intervenção psicomotora deve ser utilizada no contexto educacional, pois contribui para o desenvolvimento ideal da criança ${ }^{(21)}$.

$\mathrm{Na}$ aplicação da escala PEDro, o estudo obteve pontuação igual 4, sendo classificado com pobre em qualidade metodológica. A falta de detalhes acerca da intervenção aplicada, além da alocação não aleatorizada, o não cegamento de terapeutas e examinadores, bem como a falta de follow-up, contribuem para esse resultado metodológico ruim.

Dentro dessa perspectiva de desenvolvimento infantil, em um relato de experiência, com objetivo de analisar o desenvolvimento de crianças entre 2 e 3 anos após a intervenção cognitivo-motora, sugeriu que a intervenção psicomotora contribui para o desenvolvimento global da criança, além de verificar a melhora no desempenho motor e na socialização das crianças, de modo mais específico. O programa de intervenção aconteceu por um período de 10 encontros, que ocorriam uma vez por semana, com duração total de $3 \mathrm{~h}$ e 15 minutos por encontro, nos quais as atividades desenvolvidas foram: circuito psicomotor, coordenação motora fina e coordenação motora ampla(20).

\section{A intervenção psicomotora em pré-escolares}

O desenvolvimento psicomotor na infância busca proporcionar às crianças as condições necessárias a um bom desempenho escolar. Existem duas correntes principais de intervenção psicomotora, a psicomotricidade funcional, que segue uma linha tradicional de método dirigido objetivando o desenvolvimento das principais áreas psicomotoras, e a psicomotricidade relacional, que foca na espontaneidade, baseando-se na criatividade, nas descobertas motoras e na expressividade ${ }^{(17)}$.

A intervenção psicomotora em pré-escolares é abordada em outro estudo que verificou se a psicomotricidade relacional é mais vantajosa quando comparada com a psicomotricidade funcional, e se estas são melhores frente ao grupo controle. Desse estudo participaram 46 crianças, entre 4 e 5 anos, dividas em 3 grupos (GE- psicomotricidade relacional, GD- psicomotricidade funcional e GC- grupo controle). Os dois grupos de intervenção adotaram um protocolo de intervenção composto por 4 partes: I) ritual de entrada, II) sessão propriamente dita, III) contação de história e IV) retorno à calma, enquanto que o grupo controle realizava atividades não estruturadas. O resultado do estudo apresenta que não houve diferenças estatísticas no grupo GD em nenhum dos parâmetros avaliados (autoconceito, aptidões sociais e problemas de comportamento) em relação aos GE e GC ${ }^{(17)}$.

$\mathrm{Na}$ aplicação da escala PEDro, o estudo pontuou 7, sendo classificado com um estudo de boa qualidade metodológica. Observou-se, ainda, que a alocação não aleatorizada e o não cegamento de terapeutas e examinadores contribuíram para que o resultado metodológico apresentado não fosse melhor. 
No contexto da educação pré-escolar, um estudo teve como objetivo verificar a influência da aula de Educação Física no desenvolvimento psicomotor de alunos de 5 anos. Assim, o estudou contou com 126 participantes, de ambos os sexos, divididos em 2 grupos (GC- grupo controle e GE- grupo experimental), sendo o grupo GE assistido por um programa de intervenção estruturado em três partes ( $1^{\mathrm{a}}$ parte - aquecimento, $2^{\mathrm{a}}$ parte - fundamental com ênfase ao trabalho específico e $3^{\mathrm{a}}$ parte - retorno à calma), durante 24 semanas, com atividades realizadas uma vez por semana por 45 minutos, enquanto o grupo controle realizava intervenção não estruturada durante o mesmo período. Os resultados obtidos e comparados entre o momento de pré e pós-intervenção, através da aplicação do Teste de Avaliação Psicomotora, de autoria própria, que teve como medidas de desfecho a coordenação, o equilíbrio, o esquema corporal, a lateralidade, a organização espacial e a organização temporal, sugerem que a intervenção psicomotora estruturada realizada por educadores físicos na fase pré-escolar contribui positivamente para o desenvolvimento psicomotor da criança ${ }^{(18)}$.

Foi aplicada a escala PEDro e o estudo apresentou pontuação igual a 8, apontando, dessa forma, ser um estudo de boa qualidade metodológica, porém a ausência de cegamento de terapeutas e examinadores impossibilitaram uma melhor pontuação para o estudo analisado.

Ainda na realização de atividades psicomotoras no contexto pré-escolar, mais um estudo teve como objetivo promover o desenvolvimento neuropsicomotor integral e potencialidades individuais por meio de atividades lúdicas específicas para as crianças participantes. A intervenção psicomotora selecionou participantes pré-escolares que tinham entre 4 e 6 anos. $O$ programa interventivo teve duração de 1 ano, com atividades realizadas 2 vezes por semana, com duração de 40 minutos para crianças de 4 e 5 anos e de 50 minutos para crianças de 6 anos. Como resultado da avaliação pré e pós- intervenção, após posterior teste de organização espacial e reconhecimento do esquema corporal, o estudo apresenta que, após a intervenção proposta, as crianças apresentaram melhoras significativas quanto aos desfechos analisados, além de verificarem que existe relação entre os jogos de movimento dentro do programa de atividades e o nível de habilidades adquiridas ${ }^{(16)}$.

Na escala PEDro de avaliação metodológica, o estudo recebeu pontuação igual a 4, portanto, sendo classificado como um estudo de baixa qualidade metodológica. Observou-se que a ausência de comparações estatísticas, a não aleatorização, bem como os nãos cegamentos de terapeutas, examinadores e sujeitos da pesquisa, impediram que o estudo obtivesse uma pontuação melhor nessa escala de avaliação.

Os estudos, em sua maioria, são de baixa qualidade metodológica, segundo a escala PEDro, acarretando no baixo nível de evidência para a prática psicomotora. Além disso, foram encontrados pouquíssimos estudos na área de Ciências da Saúde, dificultando a consolidação da prática psicomotora como método de prevenção e recuperação em relação à saúde da criança, sendo esses fatores limitantes nas buscas do presente estudo. Portanto, novos estudos devem ser realizados, adotando mais rigor metodológico, além da necessidade de uma maior investigação desse tipo intervenção por profissionais da saúde, para aplicação irrestrita almejando-se bons resultados na promoção da saúde.

\section{CONCLUSÃO}

A presente revisão integrativa possibilitou a construção de uma síntese do conhecimento científico acerca da utilização da intervenção psicomotora em relação ao desenvolvimento infantil e sobre como esse tipo de abordagem contribui para a prevenção e recuperação de atrasos na pediatria. Percebeu-se que a intervenção psicomotora estruturada, apesar da heterogeneidade de protocolos adotados, apresenta resultados positivos no processo de desenvolvimento neuropsicomotor na infância, sendo um recurso passível de utilização para recuperar ou prevenir atrasos no desenvolvimento infantil.

\section{CONFLITOS DE INTERESSE}

As autoras afirmam que não houve conflitos de interesses na execução desta pesquisa.

\section{CONTRIBUIÇÕES}

Kátia Virginia Viana Cardoso e Sarah Amaral Lima contribuíram com a elaboração e delineamento do estudo; a aquisição, análise e interpretação dos dados; e a redação do manuscrito. 


\section{REFERÊNCIAS}

1. Zaqueu LCC, Teixeira MCTV, Alckmin-Carvalho F, Paula CS. Associações entre sinais precoces de autismo, atenção compartilhada e atrasos no desenvolvimento infantil. Psicol Teor e Pesqui. 2015;31(3):293-302.

2. Müller $A B$. Efeitos da intervencao motora em diferentes contextos no desenvolvimento da criança com atraso motor [dissertação]. Porto Alegre: UFRGS; 2008.

3. Spessato BC, Valentini NC, Krebs RK, Berleza A. Educação infantil e intervenção motora: um olhar a partir da teoria bioecológica de Bronfenbrenner. Movimento (Porto Alegre). 2009;15(4):147-73.

4. Moraes S, Maluf MFM. Psicomotricidade no contexto da neuroaprendizagem: contribuições à ação psicopedagógica. Psicopedagogia. 2015;32(97):84-92.

5. Almeida MHLF. O processo ensino/aprendizado através da educação psicomotora. Rev Eventos Pedagógicos. 2016;7(2):498-510.

6. Nair MK, Philip E, Jeyaseelan L, George B, Mathews S, Padma K. Effect of child development centre model early stimulation among at-risk babies - A randomized controlled trial. Indian Pediatr. 2009;(Suppl 46):s20-6.

7. Silva MG. A importância da psicomotricidade para a educação infantil [trabalho de conclusão de curso]. Campina Grande: Universidade Estadual da Paraíba; 2018.

8. Rossi FS. Considerações sobre a psicomotricidade na educação infantil. Rev Vozes Vales UFVJM Publ Acadêmicas. 2012;1(1):1-18.

9. Fonseca V. Psicomotricidade: uma visão pessoal. Constr psicopedag. 2010;18(17):42-52.

10. Fernandes JMA, Gutierres PJB Filho, Rezende ALG. Psicomotricidade, jogo e corpo-em-relação: contribuições para a intervenção. Cad Bras Ter Ocup. 2018;26(3):702-09.

11. Peruzzolo DL, Souza APR. Uma hipótese de funcionamento psicomotor como estratégia clínica para o tratamento de bebês em intervenção precoce. Cad Bras Ter Ocup. 2017;25(2):427-34.

12. Rezende LMT, Moreira OC, Oliveira-Torres J. Importância do trabalho psicomotor em aulas de educação física para pessoas com deficiência. Rev Bras Prescrição Fisiol Exercício. 2014;8(47):485-93.

13. Oliveira AC, César CPHAR, Matos GG, Passos PS, Pereira LD, Alves T, et al. Habilidades auditivas, de linguagem, motoras e sociais no desenvolvimento infantil: uma proposta de triagem. Rev CEFAC. 2018;20(2):218-27.

14. Shiwa SR, Costa LO, Costa LC, Moseley A, Hespanhol LC Jr., Venancio R. Reproducibility of the Portuguese version of the PEDro Scale. Cad Saúde Pública. 2011;27(10):2063-68.

15. Soejima CS, Bolsanello MA. Programa de intervenção e atenção precoce com bebês na educação infantil. Educar Rev. 2012;28(43):65-79.

16. Santos ACA. Psicomotricidade-método dirigido e método espontâneo na Educação Pré-escolar [dissertação]. Coimbra: Instituto Politécnico de Coimbra; 2017.

17. Costa HT, Gonçalves JFS, Pimenta FS, Arufe-Giraldez V. Influência da educação física no desenvolvimento psicomotor de crianças com cinco anos de idade. Nuances Estud Educ. 2016;27(3):79-100.

18. Ene MI, Iconomescu TH, Talaghir LG, Neofit A. Developing spatial and body schema orientation in preschoolers and primary school through physical activities. Int J Educ Sci. 2016;15(1-2):27-33.

19. Panceri C, Pereira KRG, Valentini NC. A intervenção motora como fator de prevenção de atrasos no desenvolvimento motor e cognitivo de bebês durante o período de internação hospitalar. Cad Bras Ter Ocup. 2018;25(3):469-79.

20. Brandão J, Barros M, Bessa S. Desenvolvimento psicomotor de crianças de 2 a 3 anos: proposição de atividades. Anais do Congresso de Iniciação Científica, Estágio e Docência do Campus Formosa [Internet]; 2017 [acesso em 2018 Dez 12]. Disponível em: file:///C:/Users/731088835/Downloads/10510-Texto\%20 do\%20artigo-31166-1-10-20180316.pdf

21. Mas M, Jiménez L, Riera C. Systematization of the Psychomotor Activity and Cognitive Development. Psicol Educ. 2018;24(1):38-41. 
Endereço do primeiro autor:

Kátia Virginia Viana Cardoso

Universidade Federal do Ceará - UFC

Departamento de Fisioterapia da Faculdade de Medicina

Rua Major Weyne, 1440

Bairro: Rodolfo Teófilo

CEP: 60430-450 - Fortaleza - CE - Brasil

E-mail:kvvc2004@yahoo.com.br

\section{Endereço para correspondência:}

Sarah Amaral Lima

Universidade Federal do Ceará - UFC

Departamento de Fisioterapia da Faculdade de Medicina

Rua Major Weyne, 1440

Bairro: Rodolfo Teófilo

CEP: 60430-450 - Fortaleza - CE - Brasil

E-mail: sarahamaral031@gmail.com

Como citar: Cardoso KVV, Lima SA. Intervenção psicomotora no desenvolvimento infantil: uma revisão integrativa. Rev Bras Promoç Saúde. 2019;32:9300. 